FORMATION Formation emploi

Revue française de sciences sociales

101 | janvier-mars 2008

Numéro anniversaire : Regards croisés sur les

relations formation-emploi

\title{
Les changements possibles du système français de formation continue
}

Changes in the French continuing vocational training system

Mögliche Veränderungen des Weiterbildungssystems

Los cambios posibles del sistema francés de formación continua

\section{Claude Dubar}

\section{(2) OpenEdition}

Journals

Édition électronique

URL : http://journals.openedition.org/formationemploi/1164

DOI : 10.4000/formationemploi.1164

ISSN : 2107-0946

Éditeur

La Documentation française

Édition imprimée

Date de publication : 1 janvier 2008

Pagination : 167-182

ISSN : 0759-6340

Référence électronique

Claude Dubar, «Les changements possibles du système français de formation continue », Formation emploi [En ligne], 101 | janvier-mars 2008, mis en ligne le 31 mars 2010, consulté le 30 octobre 2020. URL : http://journals.openedition.org/formationemploi/1164 ; DOI : https://doi.org/10.4000/ formationemploi.1164

(c) Tous droits réservés 


\section{Numéro anniversaire}

\section{Les changements possibles du système français de formation continue}

Claude Dubar*

La loi de juillet 1971 avait abouti à l'hégémonie du modèle de la formation professionnelle continue. L'entreprise primait sur le salarié; les petites entreprises étaient marginalisées; les inégalités étaient élevées. Qu'en est-il depuis?

Cet article a pour but de recenser et d'analyser les ruptures, inflexions, transformations changements de la formation continue «à la française », espérés par certains acteurs ou argumentés par certains chercheurs, à la suite de l'adoption de l'accord national interprofessionnel (ANI) de septembre 2003 et de la loi de mai 2004 portant l'un et l'autre sur la formation tout au long de la vie (désormais FTLV). Nous verrons que les intitulés exacts de l'accord et de la loi diffèrent quelque peu et que cette notion vient, apparemment, se substituer à celle de «Formation Professionnelle Continue (désormais FPC) dans le cadre de l'éducation permanente $(E P) »$ ainsi que s'intitulait la loi de juillet 1971, considérée comme fondatrice du système français de formation continue.

Un détour par la terminologie - et, brièvement, par l'histoire - permettra d'argumenter l'hypothèse de " trois matrices de l'éducation/formation des adultes en France» (Dubar, 2006). Elles correspondent à trois champs sémantiques différents structurant des objectifs, des configurations d'acteurs et des dispositifs de formation divers depuis les cours pour adultes, créés au XIX ${ }^{\mathrm{e}}$ siècle, jusqu'aux stages de formation en entreprise, multipliés après 1971, en passant par des formules associatives diverses comme les sessions ou les cercles (calqués sur le modèle suédois des cercles d'études) ou même les séquences d'autoformation. Les trois matrices utilisent des termes différents renvoyant à des disjonctions (éducation/ formation ; culturel/professionnel ; continu/récurrent, etc.) dont l'origine est souvent difficile à élucider, mais qui renvoient à des significations, des traditions, des modèles différents.

Quatre changements possibles peuvent alors être passés au filtre des analyses de la formation continue, en France, de 1971 à 2004, et du contexte socioéconomique actuel. Le premier concerne le passage

* Claude Dubar est sociologue, professeur émérite à l'université de Versailles-Saint Quentin en Yvelines et membre du laboratoire Printemps. II a publié récemment : La formation professionnelle continue, La Découverte, 5e édition, 2004 et Faire de la sociologie, Belin, 2006. 
potentiel d'une responsabilité de l'employeur - et du rôle-clé de l'entreprise - à la responsabilité de «l'individu » et à l'initiative du salarié, « en liaison avec son entreprise ». Il débouchera sur la question de l'accompagnement des personnes les plus éloignées du monde du travail qualifié (y compris les chômeurs) vers la formation. Le second est le basculement, évoqué par l'ANI et la loi, du cumul actuel des inégalités d'accès à la formation continue à un possible rééquilibrage au profit des plus désavantagés permettant, comme dit le législateur, un «accès effectif de tous les salariés à la formation ». Se posera la question de ce que de plus en plus d'experts appellent l'inégale « appétence » des individus envers la formation. Le troisième changement serait le passage d'un rendement très faible de la formation professionnelle continue actuelle en matière de promotion ou de salaire à un lien fort entre la formation tout au long de la vie et des parcours professionnels de mobilité. Il posera la question de la transférabilité du droit individuel à la formation (désormais DIF) et de la pratique de la validation des acquis de l'expérience (désormais VAE). Le dernier changement possible est le passage du primat actuel de l'offre de formation à la priorité aux demandes des individus : il posera les questions des relations entre la formation continue et la formation initiale et des conditions du passage de l'hégémonie des logiques d'adaptation au poste de travail à la prise en compte - notamment au niveau régional - de toutes les formes d'accompagnement des parcours des citoyens.

\section{LES TROIS MATRICES DE L'ÉDUCATION/FORMATION DES ADULTES EN FRANCE}

Comment s'orienter dans les multiples appellations : éducation/formation des adultes; formation/éducation permanente ; formation professionnelle continue ; formation continuée ; éducation récurrente ; éducation populaire ; formation post-scolaire ; auto-formation ; formation/apprentissages tout au long de la vie, etc. ? Pour y mettre un peu d'ordre, si possible ancré historiquement, j'ai proposé de distinguer trois matrices de la formation/éducation correspondant à trois champs sémantiques reliant des appellations d'objectifs, d'acteurs et de dispositifs de formation (Dubar, 2006, p. 53).

La première, centrée sur le dispositif des cours du soir, est liée à l'objectif de la promotion sociale (désormais PS) c'est-à-dire à l'accès, au moyen de diplômes ou équivalents acquis au terme d'études de type scolaire, à des positions supérieures sur l'échelle sociale. L'acteur principal est l'acteur éducatif (le Maître) qui transmet « le » savoir à des élèves (jeunes ou adultes) mais c'est aussi l'État qui finance au moins les enseignants et les institutions et, parfois (rarement), les «élèves », sous une forme ou une autre (bourse, crédit, allocation...). La formation se réalise en dehors du temps de travail mais dans un objectif professionnel : accéder, grâce au diplôme, à un niveau supérieur de qualification et donc à une promotion. On trouve cette «matrice» dans le projet de loi de Condorcet (1792) devant l'Assemblée constituante qui liait l'appellation d'« éducation tout au long de la vie », à la fois à une source de « progrès des connaissances», une garantie de "l'égalité des chances devant l'instruction » et une aide à « l'exercice de la citoyenneté ». L'égalité de tous - hommes et femmes notamment - y était fermement affirmée, en lien avec le «droit au savoir» fondé sur les valeurs républicaines (Dumazedier, 1996). Pendant longtemps, le Conservatoire national des arts et métiers a été emblématique de cette matrice. Cent soixante-dix ans après le projet Condorcet (jamais appliqué), c'est la même matrice qui sous-tend la loi Debré du 31 juillet 1959, dont les justificatifs usent des mêmes mots : ascension sociale, émancipation, valeurs républicaines, unité de la nation (Debré, 1960).

Mais, déjà à cette époque, la deuxième matrice avait commencé à se manifester. C'est celle de la formation professionnelle continue (FPC); on en trouve les prémisses dans l'Association nationale pour la formation professionnelle des adultes (AFPA) qui joue, à partir d'un modèle non-scolaire, un rôle déterminant dans la formation (terme qui émerge alors et concurrence le terme « éducation ») de la main-d'œuvre pour la reconstruction de la France d'après-guerre (Bonnet, 1999). Ce sont ensuite les impératifs de modernisation de l'économie française, d'accroissement de la productivité, de perfectionnement des cadres initiés au courant des « relations humaines » (importé des ÉtatsUnis grâce aux missions de productivité du plan 
Marshall) et, finalement, de gestion des emplois qui prennent le pas sur les objectifs de promotion, au nom de la compétitivité économique (Brucy et alii, 2007). Grâce à l'action d'une élite modernisatrice réunissant des chefs du personnel des grandes entreprises nationalisées, des hauts fonctionnaires et quelques (rares) syndicalistes (Tanguy, 2007a), la nouvelle matrice va féconder, à l'initiative de l'État (qui suscite l'ANI de juillet 1970), un nouveau système de formation à la française, celui des lois de 1966, de 1968 et surtout la loi de juillet 1971, dite loi Delors, sur la « formation professionnelle continue dans le cadre de l'éducation permanente $»$.

Cette loi réaffirme, en préambule, l'objectif premier d'adaptation des travailleurs aux changements, avant celui de promotion et, a fortiori, celui de « développement culturel » associé à l'appellation d'éducation permanente qui devient un simple «cadre». La formule des stages courts, pendant le temps de travail, d'adaptation aux changements techniques, à l'initiative de l'employeur et financés par lui, devient ainsi dominante, alors que stagnent ou régressent le nombre et le taux des adultes en « cours de promotion sociale $»$ Ces derniers représentaient plus de $30 \%$ des 1,7 million de stagiaires en 1972 et moins de $5 \%$ des 7 millions de stagiaires recensés en 2003 (Dubar, 2004).

Cette seconde matrice inscrit la formation professionnelle continue dans le droit du travail, privilégie l'obligation de financement et fait de l'entreprise le lieu central d'élaboration et d'usage de la formation. C'est l'employeur - financeur principal - qui est responsable de cette formation adaptative de son personnel. La formation continue n'a plus pour objectif premier la promotion du travail mais l'adaptation des salariés (Podevin, 1999). L'État hérite de l'objectif de promotion sociale mais il est vite concurrencé par celui d'insertion des jeunes et de réinsertion des chômeurs. En effet, la profusion, à partir de la fin des années 70, de formations pour les chômeurs, notamment les jeunes, aux résultats plus qu'incertains (Rose, 1986) a contribué au désengagement de l'État du financement des formations promotionnelles (Dubar et Gadéa, (Éds.), 1999) Quant au «cadre» de l'éducation permanente, il semble bien, rétrospectivement, constituer une coquille presque vide...
Mais quelle est donc cette troisième matrice, intitulée « éducation permanente» par la loi de 71, qui se distingue à la fois de celle de la promotion sociale et de celle de la formation professionnelle continue? L'exemple le plus parlant est sans doute celui des « cercles d'études » de Suède et du Danemark, initiés à la fin du XIX ${ }^{\mathrm{e}}$ siècle ; ils supposent l'initiative coordonnée d'individus (protestants) qui décident de se réunir pour lire (la Bible d'abord) et discuter du sens de ce qu'ils ont tous lu (grâce à la bibliothèque de prêt et à l'action d'un modérateur). Ce dispositif du cercle - bien distinct du cours et du stage - s'institutionnalise, en Suède, dès le début $\mathrm{du} \mathrm{XX}^{\mathrm{e}}$ siècle, et constitue une ressource culturelle très précieuse en matière de recyclage professionnel, d'éducation populaire ou de vie associative (Larsson, 2001; Ueberschlag et Muller, 1986). Y a-t-il l'équivalent de ce dispositif en France? Pas vraiment, même si diverses formules s'en réclament comme celle de Peuple et Culture et de son «entraînement mental » (Chosson, 1995), ou les sessions d'animation des Centres d'entraînement aux méthodes actives (CEMEA) ou, à un moindre degré, celle des Actions collectives de formation (ACF), initiées par Bertrand Schwartz en Lorraine (1994), diffusées notamment dans le Nord-Pas de Calais et rattachées explicitement par lui à un modèle d'éducation permanente (Schwartz, 1969).

Il s'agit, grâce à des "passeurs » de l'éducation populaire (Troger, 2001), de privilégier les demandes des individus associés : demande d'aides à la réalisation d'apprentissages divers liés à des problèmes de vie pratique (mécanique-auto ou coupe-couture dans les ACF en milieu ouvrier), à des questions de maîtrise et compréhension de fonctionnements techniques ou relationnels au travail, à des compétences d'animation politique ou, simplement, à des désirs de « développement culturel» (sportif, artistique, relationnel...) dans le temps libéré des loisirs (Dumazedier, 1968). Des réponses souvent associatives à ces demandes consistent en un accompagnement des apprentissages, dans des groupes où la formation mutuelle implique aussi de l'auto-formation.

Cette dernière matrice a inspiré les recommandations de l'Union européenne, notamment dans son Livre blanc de 1995, en termes de Lifelong Learning (LLL) traduit en français par « formation tout au long de la 
vie ». Fille de l'éducation permanente de l'UNESCO (Organisation des nations unis) définie, dès les années 60, comme « apprendre à être » (Faure et alii, 1972) et de l'éducation récurrente de l'OCDE (Organisation de coopération et de développement économiques) des années 70, déjà appelée «tout au long de la vie » (Forquin, 2002), cette troisième matrice reste pour l'instant un «slogan » (Méhaut, 2006). À tel point que ce sont d'autres expressions qui apparaissent juridiquement : formation tout au long de la vie professionnelle (FTLVP) dans l'ANI de 2003 et formation professionnelle tout au long de la vie (FPTLV) - associée à dialogue social - dans la loi de 2004. Ainsi non seulement «Learning» a-t-il été traduit en français par formation (et non éducation ou apprentissage) mais cette formation est associée à professionnelle. L'ancrage de la formation dans le droit du travail subsiste (c'est le Livre IX du Code du travail) et l'évolution dont il est question est «professionnelle» et non pas «culturelle». De même le dialogue social, réglementé par la loi de 2004, est-il celui qui relie les "partenaires sociaux» invités à passer d'un régime de confrontation à un régime de négociation (Tanguy, 2007).

Contrairement à la Lifelong Learning associée au développement culturel et à la citoyenneté, la formation tout au long de la vie professionnelle ou formation professionnelle tout au long de la vie «à la française » semble bien relever toujours de la matrice « formation professionnelle continu », même si elle introduit désormais des éléments de la matrice «éducation permanente». Quant à la matrice «promotion sociale», elle refait surface mais associée - nous verrons comment - à la notion de parcours professionnels.

\section{DE LA RESPONSABILITÉ DE L'EMPLOYEUR À CELLE DU SALARIÉ}

L'introduction d'un droit à la formation (DIF) va-t-il modifier « la responsabilité éminente du chef d'entreprise » (loi de 1971) sur la formation de ses salariés? Nous verrons en quoi ce «nouveau droit» reste conditionnel et en quoi son exercice dépend étroitement des incitations à et des accompagnements vers la formation, surtout des personnels les plus éloignés de l'emploi qualifié dans la grande entreprise compétitive.

\section{Individualisation ou personnalisation du droit à la formation?}

Il existe un certain consensus, parmi les représentants $\mathrm{du}$ patronat et des syndicats de salariés, comme parmi les hauts fonctionnaires, pour considérer que «l'apparition en France d'un droit individuel à la formation constitue une rupture dans l'équilibre général de notre système de formation continue» (Merle, 2006, p. 34). Ce n'est pas un hasard si, dans le texte cité, issu du colloque de Rennes, Vincent Merle aborde cinq ou six fois la question de l'autonomie des salariés: c'est un enjeu majeur que de savoir si ceux-ci - sans parler des chômeurs, des nonsalariés ou des inactifs - vont pouvoir « se saisir » de ce «nouveau droit» pour « se construire des projets professionnels », utiliser la formation pour améliorer leur condition et surtout leur «évolution professionnelle », comme le souhaitent les négociateurs de l'ANI de 2003 ou le législateur de 2004.

Dans son intervention au même colloque, Nicole Maggi-Germain se livre à un exercice d'« herméneutique juridique » (2006, pp. 103-114) pour défendre la thèse selon laquelle le DIF constitue «un dispositif plus qu'un réel droit ». En effet, il ne peut s'exercer «qu'en accord avec son employeur » (Loi 2004) ou «en liaison avec son entreprise » (ANI 2003), ce qui suppose «un accord écrit du salarié et de son employeur » (loi 2004). S'il y a désaccord, au bout de deux exercices civils consécutifs, le salarié est pris en charge au titre du CIF par le Fonds de gestion auquel cotise l'entreprise (désormais Fongécif - Fonds de gestion du congé individuel de formation). La loi précise: "Les droits acquis au titre du DIF qui n'auront pas été utilisés par le salarié ne peuvent être convertis en rémunération" (jusqu'à une nouvelle loi affirmant le contraire ?) Il s'agit donc, d'un point de vue juridique, d'un droit « conditionnel et conditionné ». Pas d'un droit «réel» qui «s'incorpore au patrimoine du salarié ». Ni d'un droit «personnel» qui supposerait qu'un salarié « puisse exiger qu'on lui fournisse une formation ». Tout ceci limite l'autonomie des salariés néanmoins appelés à être « acteurs de leur formation ». 
C'est ce qui fait écrire à la juriste que, contrairement à la loi de modernisation sociale de janvier 2002 créant la validation des acquis de l'expérience (droit $d e$ chaque personne de faire valider ses acquis), la loi de mai 2004 ne crée qu'un droit à l'accès des seuls salariés, munis d'un contrat de travail, à condition d'avoir l'accord de l'employeur. Il dépend donc des conditions de la relation salariale: initiative du salarié, transaction avec la hiérarchie, accord formel de l'employeur. Qu'une de ces conditions ne soit pas remplie et le droit ne peut s'exercer... D'où l'hypothèse qu'il s'agit plus d'une «individualisation» de la responsabilité de se former, de «maintenir son employabilité » pour le salarié que d'une "personnalisation » d'un nouveau droit fondamental de l'homme (Maggi-Germain, 2004). Même si «rien n'est joué » et que l'usage peut le tirer vers cette seconde hypothèse, le DIF n'est pas un nouveau droit personnel ouvert à tous les citoyens.

\section{La question de l'accompagnement du « salarié acteur»}

Il est vrai que l'ANI de 2003, en partie relayé par la loi, prévoit toute une batterie d'outils de gestion pour rendre ce droit moins formel pour les salariés : entretien annuel avec le supérieur hiérarchique, bilan de compétence avec congé ad hoc, passeport formation à usage exclusif du salarié, formation hors travail reconnue et indemnisée à hauteur de la moitié du Smic (salaire minimum interprofessionnel de croissance), formation sur le temps de travail possible en cas d'accord de branche ou d'entreprise et, surtout, « engagement écrit de l'employeur formalisant la prise en compte des acquis de la formation ». $\mathrm{Ne}$ s'agit-il pas là de «marges d'autonomie pour les salariés qui peuvent enfin devenir acteurs de leur formation » ( $c f$. Iforep, p. 7)? Dans un numéro spécial de sa revue où s'expriment des représentants des principaux « partenaires sociaux » (CGT, CFDT, CFTC, CGT-FO, MEDEF...) $)^{1}$, l'Iforep, organisme de formation des Caisses d'action sociale d'EDF (Électricité de France) vérifie qu'un consensus semble se

\footnotetext{
${ }^{1}$ Respectivement Confédération générale du travail, Confédération démocratique du travail, Confédération des travailleurs chrétiens, Force-ouvrière, Mouvement des entreprises de France.
}

dessiner sur "l'émergence, dans l'entreprise, d'une nouvelle relation à la formation» (Idem, p. 8). En tous cas dans une grande entreprise comme EDF qui s'est dotée, depuis longtemps, d'un marché interne $\mathrm{du}$ travail et pratique le « dialogue social» avec des syndicats relativement «puissants ». Mais dans les autres ?

L'enquête complémentaire à l'enquête Emploi de 2000, associant à l'INSEE (Institut national de la statistique et des études économiques), le CÉREQ (Centre d'études et de recherche sur les qualifications) et la DARES (Direction de l'animation de la recherche, des études et des statistiques) et effectuée auprès d'un échantillon de 28700 personnes de 15 à 65 ans (désormais dénommée FC 2000), montre à quel point l'initiative de l'employeur reste décisive pour l'accès à la formation professionnelle continue en France. Le poids de l'initiative personnelle (formations décidées par «vous-même exclusivement») reste modeste parmi les $28 \%$ de personnes déclarant avoir suivi une formation entre janvier 1999 et février $2000: 37,7 \%$ des « formés » mais seulement $21,1 \%$ des ouvriers et employés passés en formation. Beaucoup de personnes classées dans cette grande catégorie (qui concerne les deux tiers des salariés) non seulement n'ont pas suivi de formation durant cette période $(88,2 \%$ des ouvriers non qualifiés) mais se disent non informées de l'obligation de financement de l'employeur, de l'existence d'un plan de formation ou du CIF (près des trois quarts des ouvriers non qualifiés). Comment rendre ces salariés, souvent classés «sans qualification», travaillant souvent dans des PME (petites et moyennes entreprises) ou sur des contrats dits atypiques (temps partiel, CDD - contrat à durée déterminée -, intérim...), «acteurs de leur formation et de leur parcours professionnels »?

Il paraît assez évident que, pour eux en tous cas, " tout dépendra de l'accompagnement du DIF au sein des entreprises, branches, marchés de l'emploi» (Merle, 2006, p. 35 ; Méhaut, 2006, p. 166). Si aucune mesure d'incitation n'est prise, si le remplacement pendant la formation n'est pas assuré, si des perspectives d'amélioration du salaire ou de l'emploi ne sont pas ouvertes, si aucune aide à la rédaction de projet n'est apportée, on ne voit aucune raison pour que ces salariés se manifestent 
spontanément pour bénéficier d'un DIF qui n'est qu'un droit conditionné à l'accord de l'employeur. S'il n'y a aucun médiateur entre ce dispositif et ces salariés (qu'il s'agisse de la hiérarchie, du délégué syndical, de professionnels ou de proches), on ne voit pas bien comment et pourquoi ils se manifesteraient comme volontaires. Le mot même de formation a tellement été, chez certains d'entre eux, dans le passé (et toujours aujourd'hui), associé à celui de « plan social», que se manifester serait prendre le risque de se faire remarquer - stigmatiser - comme susceptible de faire partie de la prochaine liste (appelée souvent «charrette») de "salariés... en mobilité externe »...

Le changement consistant à faire de chaque salarié un acteur de sa formation suppose une mobilisation collective pour ouvrir des perspectives positives à tous ceux qui n'en ont pas. Est-ce ce qu'ont voulu les signataires de l'accord ou les votants de la loi ? Pas sûr. Derrière le consensus apparent se cachent des divergences profondes sur le sens véritable à accorder au DIF : s'agit-il, avant tout, de déplacer la responsabilité de la non-formation du chef d'entreprise vers les salariés eux-mêmes (jusqu'à en faire, un jour, une faute professionnelle) ou de faire avancer un nouveau droit de formation continue (comme le droit de l'instruction, de la santé ou... du logement) qui deviendrait, un jour, opposable par les salariés exigeant une formation qualifiante ou une validation de leur expérience personnelle? Entre "une conception libérale de l'investissement en capital humain» (Méhaut, 2003, p. 29) et une «conception élargie de la formation articulant les diverses logiques de formation» (Méhaut, 2006, p. 166), les acteurs institutionnels étaient et restent manifestement partagés. Le «nouveau dispositif» est, en fait, un compromis permettant à chaque « décideur» d'y projeter son propre vocabulaire : flexibilité, employabilité, adaptation et mobilité accrues pour le patronat, marges de manœuvre salariales, reconnaissance des formations et sécurisation des parcours professionnels pour les syndicats (De Calan et Quentin, 2006). Quant aux « experts», ils sont partagés entre le constat d'un « verre à moitié vide» (pas de droit à part entière) et l'espoir d'un « verre à moitié plein » (engagement de lutte contre les inégalités).

\section{RÉDUIRE LES INEGALITÉS?}

\section{De la reproduction des inégalités à un accès accru des plus démunis?}

Quelle que soit la source statistique utilisée (traitement des déclarations obligatoires des formations par les employeurs, dite 24 83, par le CÉREQ, enquêtes FQP de l'INSEE, enquête FC2000...), depuis l'entrée en vigueur de la loi de 1971, les résultats concernant les inégalités d'accès à la formation continue sont quasiment toujours les mêmes depuis les années 70 jusqu'aux années 2000 (Dubar, 2004) : les chances d'accès à la formation continue des ouvriers et employés non qualifiés (16,4\% en 2000) sont trois fois plus faibles que celles des ingénieurs et cadres $(52,8 \%$ en 2000$)$ et prés de deux fois moindres que celles des ouvriers ou employés qualifiés (29,1\% en 2000). En 2006, pour la seule formation financée par les employeurs, les taux d'accès étaient les suivants: ouvriers $31,2 \%$, employés $32,9 \%$, techniciens et agents de maîtrise, 55,8 \%, ingénieurs et cadres 54,4\% (source CÉREQ, non publiée). Les ingénieurs, cadres, techniciens étant déjà les plus diplômés et les plus qualifiés, les expressions utilisées pour désigner cette inégalité « structurelle » sont frappantes: «la formation va à la formation» (Fournier 2006, p. 30), «l'effet Matthieu» (« car on donnera à ceux qui ont déjà » Évangile selon Saint Matthieu; Möbus 2006, p. 68) ou encore la «reproduction des inégalités sociales 》 (Fritsch 1971 ; De Montlibert, 1991). Serait-ce une sorte de loi sociologique ou de fatalité sociale?

On peut argumenter qu'il n'en est rien grâce à une analyse multivariée intéressante: «Les inégalités catégorielles d'accès diminuent avec l'effort de formation des entreprises » (Aventur et Hanchane, 1999, p. 6). Le suivi d'un panel d'entreprises entre 1984 et 1992 le montre: les inégalités sont maximales au sein des entreprises de moins de 10 salariés et des secteurs sans marché interne ni gestion des ressources humaines, il est minimum au sein des entreprises de plus de 2000 salariés dans des secteurs à gestion active de la main-d'œuvre. Ce qui fait dire aux auteurs de l'article cité : "la discrimination positive à l'égard des moins qualifiés est possible.» (Idem, p. 12) Il existe, dans les entreprises les moins 
inégalitaires, «des procédures équitables garantissant l'égalité des chances d'accès", par exemple la consultation annuelle du Comité d'entreprise sur le plan de formation et sa réalisation, la garantie d'un droit à la qualification (accès au niveau $\mathrm{V}$, soit niveau $\mathrm{CAP} / \mathrm{BEP}$ pour tous ceux qui ne l'ont pas), la stratégie volontaire de la direction d'entreprise et donc «le choix délibéré en matière d'allocation des ressources de formation » (Idem, p. 15). C'est à ces conditions que «la croissance de l'effort va bien de pair avec une inégalité moindre» (Idem, p. 18). On pourrait montrer que le même type de raisonnement peut s'appliquer aux inégalités sexuées et aux inégalités de classe d'âge, sachant que "l'accès à la formation est surdéterminée par le niveau de qualification » (Fournier, 2003, p. 43). Le préalable à la réduction de ces inégalités est bien un changement volontariste des entreprises rééquilibrant les inégalités (de salaire et de perspectives de carrière) entre hommes et femmes et entre jeunes et vieux, par exemple en permettant le rapprochement des calendriers masculin et féminin de carrière ou en luttant contre la marginalisation des salarié(e)s âgé(e)s (Idem, p. 44).

Mais cette équité, accompagnée d'une égalisation des chances (« offrir des opportunités accrues à ceux qui n'en ont pas eues $»)$, ne garantit pas l'égalité des résultats (même dans les grandes entreprises ayant une stratégie volontariste, les inégalités ne s'inversent pas). Même des expériences comme les Actions collectives de formation, animées d'une volonté égalitaire, se heurtaient à d'incompressibles inégalités entre individus de même classe ou couche sociale (Hédoux, 1982). Pour les comprendre, il faut, à coup sûr, « entrer dans la boîte noire des comportements individuels». Or ceux-ci obéissent à des «motivations » qui ne placent pas nécessairement la formation parmi les priorités, même lorsque des opportunités apparaissent. Pour «se former» et non « être formé » (sur le poste de travail comme dans la FEST, - Formation en situation de travail décidée par l'employeur), il faut, selon les termes de l'accord de 2003, une... «appétence» pour la formation. De quoi s'agit-il ? D'où vient cette notion nouvelle? À quoi renvoie-t-elle? Des travaux ont commencé à nous éclairer sur ce point.

\section{Peut-on parler d'« appétence » en matière de formation?}

Cédric Frétigné (2007) s'est efforcé de traquer l'apparition de ce terme dans des documents d'experts préparatoires aux accords (ceux qui ont échoué en 2001 et ceux qui ont été conclus en 2003). Il en tire deux conclusions qui, contrairement à ce qu'il laisse supposer, ne sont pas nécessairement liées entre elles. La première, c'est que la notion d'appétence a constitué un outil de consensus entre les représentants patronaux et syndicaux : au nom de la « responsabilisation » et de « l'individualisation», le fait d'aller en formation «à son initiative propre » a été imputé au «désir», à «l'envie», bref à l'appétit pour la formation du salarié, devenue, dans l'air du temps, «appétence » (la notion est utilisée par le marketing qui calcule des indices d'appétence par la probabilité d'achat d'un produit). Un dicton de chez moi dit: «on peut conduire un âne à l'abreuvoir, on ne peut pas le forcer à boire ». Cette métaphore physiologique qui fait de la formation une marchandise vitale suppose une soif d'apprendre au moins potentielle. Mais, et c'est la seconde conclusion de Frétigné, les introducteurs de cette notion ont été influencés par les théories psychologiques du management, et notamment celles qui inspirent la «pyramide des besoins» de Maslow. Selon ce modèle, les besoins de type «culturel» ou liés à la « reconnaissance de soi », bref «secondaires» ne peuvent émerger et devenir conscients que lorsque les besoins de type "physiologique» ou "sécuritaire », bref «primaires» (revenu décent, santé, logement) sont satisfaits. La formation volontaire ferait partie de ces «besoins de second type » qui, pour s'exprimer, supposent satisfaits les désirs, envies, appétits dits primaires. Ceux qui ne boivent pas à l'abreuvoir du savoir seraient-ils simplement les plus nécessiteux? Je ne pense pas que ce soit l'opinion de ces experts médiateurs qui a fortement contribué à faire adopter la notion d'appétence. Mais il est possible que cette interprétation ait contribué à « débloquer» la méfiance de certains des « partenaires ».

Sur la base des réponses à l'enquête FC 2000, Christine Fournier $(2004,2006$, pp. 25-40) a tenté d'en avoir le cour net. Puisqu'avec le vote du DIF, 
«désormais tout salarié pourra bénéficier d'au moins $20 \mathrm{~h}$ de formation par an cumulables sur 6 ans » (sauf s'il est à temps partiel et n'en bénéficie qu'au prorata des heures travaillées), il faudra qu'il montre «s sa détermination à se former », qu'il donne des témoignages de « son engagement en formation », qu'il manifeste «son intérêt pour le développement de ses compétences ». Toutes ces expressions seront désormais considérées par les experts comme des « signes d'appétence» pour la formation. Or, il existe dans le questionnaire de l'enquête FC 2000 des questions sur les «besoins non satisfaits de formation » permettant, par exemple, de saisir, au cours des 14 mois précédant l'enquête, les éventuels désirs non satisfaits de formation ou d'anticiper et évaluer la force des besoins futurs. Le raisonnement de la chercheuse est simple : si le non-accès est dû à un défaut de la soi-disant «appétence», ceux qui n'ont pas suivi de formation ne devraient pas non plus déclarer des « besoins non satisfaits ».

Or ça n'est pas vraiment ce qu'on observe. Les résultats sont plus compliqués et intéressants. Par exemple, chez les ouvriers, $19 \%$ des «formés» (ceux qui ont déclaré avoir suivi au moins une formation en 1999) répondent avoir eu un besoin de formation non satisfait contre $16 \%$ des «non-formés». L'écart est faible, peut-être statistiquement non significatif. Par ailleurs, $47 \%$ de l'ensemble des ouvriers répondent positivement à l'item sur les «besoins futurs assez ou très forts $\gg$ contre $68 \%$ des professions intermédiaires et $67 \%$ des cadres. L'écart demeure mais moins grand qu'en matière de taux d'accès. Enfin et surtout, "la moitié des ouvriers et employés se disent motivés par un changement d'emploi, un diplôme ou la reconnaissance d'une qualification contre $16 \%$ des cadres » (Idem, p. 31). Ainsi « la réponse à la question des besoins dépend intimement des représentations que les salariés se font de la formation et de leurs perspectives d'emploi ». En fait, les variables structurelles (taille et secteur de l'entreprise, présence d'un marché interne et de chances de promotion) qui pèsent plus que les variables individuelles (âge, sexe, PCS profession et catégorie sociale...) sur l'accès à la formation (Dubar, 2004) pèsent encore plus sur "l'inégale appétence des salariés envers la formation » (Fournier, 2006). C'est bien «le contexte professionnel d'une part, les finalités poursuivies de l'autre " (Fournier), et donc «l'articulation entre la trajectoire subjective et le système d'action » (Dubar, 2006) qui rendent compte à la fois de l'accès effectif (Dubar) et des souhaits d'accès à la formation (Fournier). Dans tous les cas, comme l'écrit C. Fournier (et comme l'écrivait déjà B. Schwartz, il y a quarante ans) « la formation n'est pas une fin en soi : le projet de se former suppose un intérêt à se former ». Si c'est cela, l'appétence, alors il fallait l'écrire clairement: pas de formation sans résultat espéré pour celui qui se forme.

\section{LA FORMATION PROFESSIONNELLE CONTINUE... ET APRÈS ?}

\section{Quel impact sur les salaires et la mobilité ?}

Tous les avis d'experts et toutes les analyses de chercheurs se rejoignent (pour une fois) pour constater (et souvent déplorer) le «faible rendement des actions de formation continue aussi bien pour les salariés (salaires, carrières...) que pour les chômeurs (retour à l'emploi) » (Dayan et Eksi, 2007). Une analyse économétrique appliquée aux données individuelles conclut à l'existence d'un «biais de sélection empêchant de décider si c'est la FPC qui améliore les salaires, promotions ou accès à l'emploi ou si c'est le niveau de ces derniers qui détermine l'intensité de l'effort de formation $»$. Malgré ce biais, il faut reconnaître que «la FPC à la française est très peu rentable en terme financier» et "qu'elle n'a pas d'impact significatif sur les carrières salariales dans les entreprises formatrices ». Tout au plus permetelle de «se maintenir en emploi en actualisant ses compétences » et, parfois d'éviter une « «démotion » [inverse d'une promotion] voire une marginalisation de l'emploi » (Fougère, Goux, Maurin, 2001).

Les résultats de l'enquête FC 2000 et des enquêtes FQP vont dans le même sens que les enquêtes précédentes: seulement $5,5 \%$ des répondants «formés dans l'année 1999 » mentionnent une augmentation de salaire obtenue grâce à la formation suivie. Ce taux n'a fait que diminuer, comme celui 
des « promus grâce à la formation ». Mesuré à partir de l'enquête FQP 1993, il était de 9,4\% pour les $31,5 \%$ de la population active qui avaient suivi une formation dans les cinq ans précédant l'enquête. Les analyses issues des deux enquêtes se rejoignent: "l'influence salariale de la formation est de faible ampleur sauf lorsqu'elle intervient de façon répétée » (Dupray et Hanchane, 2001, p. 54). Depuis longtemps, la FPC à la française sert en fait, avant tout «à fidéliser, sélectionner les salariés les plus performants » et, ainsi, "à creuser les écarts en capital humain entre les moins diplômés et les plus diplômés au lieu de les réduire» (Idem). Elle sert aussi à « améliorer l'insertion des jeunes embauchés dans l'emploi », ce qui ne signifie pas, en tous cas pour les ouvriers, les promouvoir ni même leur permettre de s'inscrire dans des filières d'évolution (Poullaouec, 2004). Les effets les plus mentionnés par les répondants de FC 2000 sont les suivants ; 1/se former à une technique $(70 \%) 2 / \mathrm{s}$ 'adapter à son poste de travail (58\%) 3/mieux connaître l'entreprise (21\%) $4 /$ se remettre à niveau $(15,5 \%)$. Les conclusions de l'analyse économétrique sont confirmées : " un stage de formation continue diminue le risque de départ de l'entreprise: de 30 à $36 \%$ après passage en FPC » (Idem, p. 62). Quant au lien entre le suivi de formations et les parcours professionnels ascendants, il ne demeure que pour une minorité de cadres promus, gros consommateurs de formations, masculins, jeunes (moins de 35 ans) et déjà diplômés à bac +3 dans les métiers des services aux entreprises et de la finance (Gadéa et Trancart, 2003, p. 109).

Ces constats doivent être reliés à des éléments d'histoire; lors de la négociation des accords de 1970, les syndicats - notamment la CGT dont c'était un objectif explicite - avaient échoué dans leur tentative de faire « reconnaitre les qualifications acquises en formation continue ». Le CNPF (Centre national du patronat français) avait été à l'offensive - créant des centaines de postes de permanents affectés à la formation - pour faire de la FPC « un investissement rentable ", ce qui signifiait des salariés mieux formés, mieux intégrés à l'entreprise, plus performants, sans aucune obligation de reconnaissance. Il aura fallu une dizaine d'années pour que la FPC devienne ainsi un «outil de management» dans les grandes entreprises actives en matière de gestion des ressources humaines. Des innovations de formation ont remplacé les formations « ouvres sociales» et accompagné la modernisation et le passage d'une logique négociée de la qualification à une logique individualisée des compétences (Dubar, 2004). La rubrique "promotion» est devenue de plus en plus rare dans les statistiques des formations à l'initiative des entreprises comme dans les statistiques des formations «à initiative personnelle 》 (Dubar et Gadéa (Éds.), 1999) La formation dans les entreprises «modernisées» est devenue un élément de l'organisation du travail et de la gestion de l'emploi (Podevin et Verdier, 1989). Compétences des salariés «mobilisés» et compétitivité de l'entreprise « apprenante » allaient de pair dans cette dynamique modernisatrice. Les syndicats étaient marginalisés et la FPC instrumentalisée.

L'accord de 2003 introduit un changement potentiel d'importance. Dans son article 8, il précise: "l'entreprise définit, avec le salarié, avant son départ en formation, la nature des engagements auxquels elle souscrit [...] le salarié accède en priorité dans un délai d'un an à l'issue de sa formation, aux fonctions disponibles correspondant aux compétences acquises [...] il se voit attribué la qualification correspondant à l'emploi occupé ». Pas besoin d'une longue exégèse pour apprécier les avancées et les limites de cet engagement. La formation suivie (dans le cadre du Plan de formation) peut être reconnue par l'entreprise et déboucher sur une promotion professionnelle. Mais celle-ci est conditionnelle (« en priorité» «fonctions disponibles» " correspondant aux compétences ») et non transférable à une autre entreprise. Et qui décidera de la « correspondance» entre « compétences acquises et fonctions disponibles $»$ ?

Entre la simple obligation faite aux salariés «d'entretenir leur employabilité» en «adaptant leurs compétences » et la promesse garantie d'une mobilité professionnelle ascendante par la formation, la nouvelle législation insiste, pour les salariés, sur « la meilleure maitrise de leur évolution professionnelle » (Préambule de l'accord) ou sur «de meilleurs parcours professionnels » plus sécurisés « de façon à ce que la mobilité nécessaire puisse être choisie» (Exposé des motifs de la loi). Articuler la nouvelle 
formation (professionnelle) tout au long de la vie (professionnelle) aux évolutions professionnelles apparait bien comme l'objectif principal et l'innovation essentielle des nouveaux dispositifs. Que recoupent-ils au juste? Se résument-ils aux mobilités internes conditionnelles précédentes? S'agit-il encore et toujours de sélectionner ceux et celles qui ont fait preuve de leur «identité d'entreprise» (Dubar et Engrand, 1991) au détriment des autres formes identitaires?

\section{Des reconnaissances et des validations transversales?}

La réponse aux questions précédentes dépend de ce que les acteurs feront des nouveaux dispositifs, et notamment de la validation des acquis de l'expérience (loi de janvier 2002) qui permet à quiconque de monter un dossier et de le défendre devant un jury pour obtenir une certification reconnue. Ce nouveau droit peut être jumelé avec le DIF ou la période de professionnalisation prévue par la loi de 2004. Il peut aussi revaloriser la formation des chômeurs dont l'image, par suite de la trop grande proportion de « stages parkings » ne débouchant ni sur l'emploi ni sur le diplôme, resta longtemps mauvaise mais qui pourrait se modifier avec la mise en œuvre du Plan d'action de retour à l'emploi (Tuchszvier, 2002). La VAE peut aussi s'appliquer sans aucun passage en formation (formelle), ce qui manifeste «une réelle évolution des conceptions françaises de la formation professionnelle» (Méhaut, 2006, p. 166). Pour la première fois depuis longtemps, en France, la professionnalisation et sa reconnaissance ne passent plus par des situations formatives classiques : l'expérience est source de compétences validables. Les certifications recensées dans le Répertoire national de la certification professionnelle sont reconnues sur tout le territoire. Une transversalité est donc théoriquement possible sur la base d'une mise en valeur de l'expérience, c'est-à-dire de réalisations de toute sorte (et plus seulement professionnelles) assurant le lien argumentaire entre performances atteintes (empiriquement fondées) et compétences supposées (reconnues par le jury).

Il reste à passer du « slogan » de la formation tout au long de la vie, et de ses innovations formelles (VAE, DIF, contrat et période de professionnalisation) aux «pratiques» qui restent très marquées en France par le poids considérable du "modèle scolaire» (Méhaut, 2006, p. 164). Car si l'élévation générale spectaculaire du niveau de formation initiale peut théoriquement favoriser les apprentissages ultérieurs, la représentation selon laquelle «tout se joue à l'école » et que «le diplôme obtenu en formation initiale détermine toute la carrière " constitue un «frein considérable à la reconnaissance de toutes les autres formes d'apprentissage ». Certes l'alternance existe - y compris sous statut scolaire ou universitaire - et se développe, mais la hiérarchie formation générale/formation technique/formation professionnelle et la sélection par l'échec qui la structure sont tellement prégnantes qu'elles constituent des obstacles majeurs à la reconnaissance à part entière, en France, des certifications de la formation continue. Ces obstacles sont-ils pour autant insurmontables?

\section{DU PRIMAT DE L'OFFRE DE FORMATION À LA RÉGULATION PAR LA DEMANDE}

\section{Vers une nouvelle configuration d'acteurs?}

Contrairement à la France, l'Allemagne, grâce notamment au système dual (Duales System) et au rôle des Syndicats et des Régions (Lander), possède, depuis longtemps, "une filière qualifiante, institutionnalisée, articulant formations continues et positions socioprofessionnelles 》 (Möbus, 2006, p. 66). Alors qu'elle partage avec la France "l'importance de la formation formelle sur le marché du travail, sanctionné par un titre officiel» et «le rôle primordial de la formation initiale pour préfigurer le parcours ultérieur ", l'Allemagne a su lier formations continues et progression professionnelle. L'accès à la formation continue diplômante et reconnue par les entreprises y est nettement facilitée par la dissociation entre la classification des diplômes de formation générale et celle des diplômes professionnels, du brevet d'apprentissage au diplôme d'ingénieur tous accessibles en formation continue et régulés par la négociation sociale, dans les Lander. 
Les demandes des salariés sont premières et prises en compte ou non au terme de négociations paritaires aboutissant à des formations financées par le budget de l'État fédéral.

L'accès individuel aux formations continues, l'expérience professionnelle acquise et les modes de régulation collective liant ces formations aux parcours professionnels au niveau «régional» forment système dans le cas allemand, pas dans celui de la FPC française. Les écarts entre les taux de participation à la formation formelle (celle qui est certifiée) des diverses catégories d'adultes ( 25 à 64 ans) diffèrent en France et en Allemagne : en 2003, cinq fois plus d'actifs occupés $(2,6$ contre $0,5 \%)$ et six fois plus d'inactifs $(6,2$ contre $1,9 \%)$ suivaient ces formations volontaires en Allemagne. En France, «le dispositif FPC a saturé la quasi-totalité de la formation continue » alors qu'en Allemagne "l'étalement de la formation certifiée au-delà de l'entrée dans la vie active tend à produire une distribution plus uniforme des diplômes par génération» (Möbus, 2006, p. 71).

Il est intéressant de comparer ce système avec celui des Fongécif, récemment étudiés par une équipe de recherche à partir de la comparaison de trois monographies dans trois régions (Podevin et Ghaffari, à paraître en 2008). Rappelons que le CIF est un droit conditionnel permettant à des salariés de suivre, à titre individuel, des formations permettant « d'accéder à un niveau supérieur de qualification, de changer d'activité ou de profession, de s'ouvrir plus largement à la culture et à la vie sociale » (Code du travail). Les demandes individuelles sont traitées régionalement par les Fongécif où cotise l'employeur et qui sont paritaires. Elles sont acceptées (en 2005, 65\% des 52513 demandes à comparer avec les six millions de " formés » par les plans de formation) «si elles sont sélectionnées à l'aune de critères communs à l'ensemble $d u$ Fonds ». Le principal critère étant le réalisme professionnel d'un projet dans le cadre d'un parcours pertinent et cohérent (Idem, p. 7). La principale conclusion de la recherche est que chaque Fongécif est devenu "producteur de norme applicable aux demandes de formation ». À travers les qualités attendues d'un dossier se construisent des règles de sélection qui sont des types de compromis entre "principes d'équité» (égalité formelle ou discrimination positive) et «adéquation au système productif local » (statique ou dynamique). Ainsi se met en place, à petite échelle, ce qui pourrait préfigurer un mécanisme de régulation fondé sur les demandes, à l'échelon régional, examinées par des experts (les conseillers ont un rôle déterminant), vérifiant l'adéquation à des priorités régionales décidées par les élus. On peut légitimement penser que le cadre régional est un choix politique porteur d'avenir, surtout s'il s'accompagne de financements publics ciblés sur les demandes des plus éloignés du monde du travail et surtout de l'emploi qualifié.

\section{La faible articulation formation initiale/formation continue}

Cette forte spécificité du système français de formation continue peut-elle changer? On sait que Jacques Delors comptait sur la FPC pour «faire bouger » le système scolaire et qu'il fut très déçu du faible impact de l'une sur l'autre (Delors, 1991). Vincent Merle va jusqu'à parler d'hostilité de beaucoup d'enseignants de formation initiale à l'égard d'une formation continue qui constitue pour eux comme un autre univers (Merle, 2004, p. 131). Il est incontestable que très peu d'élèves ou d'étudiants ont été informés, à l'école, de l'existence d'opportunités de la formation tout au long de la vie, au-delà de celles de la formation professionnelle initiale. Tout se passe comme si toute la carrière professionnelle se jouait, en France, à l'école et à l'université, avant 25 ans, selon le niveau et la spécialité du diplôme et de la filière (grandes écoles, universités, autres). Le constat fait par Fritsch (1971) de la marginalité de la formation continue par rapport à la formation initiale serait-il toujours vrai, près de quarante ans plus tard ? De fait, la quasi-hégémonie de la «FPC financée par l'employeur», consistant en stages courts (moins de $40 \mathrm{~h}$ par an) d'adaptation au poste de travail ou d'initiation aux techniques (informatique, langues, mécanique, etc.), n'a pas facilité le rapprochement de la formation continue avec la formation initiale. Et pourtant des analystes notent, depuis une trentaine d'années, grâce à la professionnalisation des formations et à la diversification des formes de formations continues (Hanchane et Lambert, 2003), un vrai rapprochement entre les écoles (certaines, du moins) et les entreprises (idem), entre des enseignants professionnels et des 
professionnels d'entreprise (Gonnin-Bolo, 2005). Comment comprendre ce paradoxe ?

Pour tenter de le faire, en éclairant la diversité des attentes en matière de formation, je m'appuierai sur une recherche très approfondie sur des publics du Conservatoire national des arts et métiers (CNAM) qui joue un rôle d'interface, depuis longtemps, entre formation initiale et formation continue (Correia et Pottier, 2000). Alors que, jusqu'aux années 70-80, le CNAM restait fixé sur son modèle traditionnel « scolaire» de promotion sociale en cours du soir visant le diplôme d'ingénieur, il a tenté de répondre à la diversité des attentes de ses auditeurs. C'est ce que la recherche a tenté de comprendre en dégageant les logiques plurielles de ces auditeurs qui ont des rapports différents aux savoirs (et non au Savoir) et à l'École. Pour comprendre ces logiques, ils ont dû reconstituer des types de parcours, tant il est à nouveau vérifié que c'est bien le lien le plus éclairant. Un seul type de parcours de "promotion sociale» (ascendant, volontariste, de type scolaire) est centré sur les savoirs théoriques et concerne des jeunes, ayant au moins bac +2 , masculins au deux tiers et déjà membres des professions intermédiaires (techniciens, informaticiens, conseillers). Ce sont des jeunes diplômés pourvus d'une «identité de réseau» (Dubar et Engrand, 1991). Un autre parcours type, baptisé "gestion rationnelle», est opportuniste, pragmatique, fait de saisies d'opportunités de changement d'emploi, centré sur les savoirs d'action et concernant des salariés pourvus d'une «identité d'entreprise » (Idem). Un dernier parcours concerne des plus âgés, une forte minorité de femmes et de personnels administratifs : il est appelé « gestion sous contrainte » et vise une stabilisation dans l'emploi ; il est centré sur les savoirs pratiques et le court terme. Concernant des salariés dotés d'une «identité catégorielle » (Idem), ces derniers parcours typiques sont les plus menacés, combinant de faibles ressources avec les durées de formation les plus courtes. Selon les auteurs de la recherche, «c'est la cohérence entre le type d'objectif, l'importance des ressources et la longueur du cursus » qui permet de comprendre les configurations des relations entre formations et parcours professionnels et donc les rendements différentiels des formations (Correia et Pottier 2000, p. 80). Seule une configuration inscrit la formation continue dans le prolongement direct de la formation initiale, les autres sont ancrées dans des problématiques de résolution de problèmes, distinctes de celle de la formation initiale.

C'est parce que le CNAM a changé sa politique et relativisé son modèle scolaire qu'il peut construire des réponses de formation à partir d'une analyse compréhensive des demandes des adultes. Ce n'est plus l'offre qui impose sa conception du Savoir, ses critères d'évaluation, ses filières de formation à des " élèves ", ce sont potentiellement les demandes des « auditeurs », leurs logiques diversifiées, leurs savoirs valorisés, leurs perspectives d'avenir qui suscitent des réponses en termes de formation mais aussi d'accompagnement et de valorisation de l'expérience. La prise en compte de la diversité des apprentissages et des rapports aux savoirs divers et légitimes se substitue à l'inculcation de «savoirs-objets» (Charlot, 1997) renforçant et légitimant la sélection sociale. C'est la reconnaissance de la diversité «démocratique » qui prend la place de l'uniformité « républicaine ». C'est la proximité des procédures décentralisées de traitement des demandes et la pertinence des réponses des offreurs et des décideurs politiques qui peuvent faciliter, petit à petit, un changement majeur de paradigme en matière de formation continue.

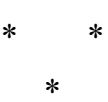

La loi de juillet 1971 avait abouti à l'hégémonie de la matrice « formation professionnelle continue » sur le système français de formation continue. L'entreprise et ses contraintes primaient sur le salarié et ses «besoins"; les non-salariés ou les salariés des petites et moyennes entreprises étaient marginalisés ; les inégalités se maintenaient à un haut niveau; la rentabilité des formations pour les stagiaires était faible et les offres des organismes, en lien plus ou moins étroit avec les «besoins des entreprises» primaient sur les demandes individuelles. Les matrices «promotion sociale» et «éducation permanente » étaient dominées, presque éliminées.

Les dernières législations (loi sur la modernisation sociale de 2002, loi sur la formation tout au long de la vie professionnelle de 2004) visent à rééquilibrer ces trois matrices. Si la formation professionnelle 
continue demeure dominante (ancrage dans le droit du travail, financement par les entreprises), des éléments relevant de l'éducation permanente ont vu le jour (validation des acquis de l'expérience, droit individuel de formation) et certaines mesures relevant de la promotion sociale sont présentes (liens avec les parcours professionnels). Mais l'avenir dépend des conditions et de l'issue des quatre dynamiques abordées dans cet article : l'accroissement de la responsabilité (individuelle et collective) des salariés; la diminution des inégalités (de catégorie, genre et âge) d'accès à la formation; l'amélioration visible des effets des formations suivies, notamment sur les mobilités ascendantes ou les retours à l'emploi ; la priorité accordée aux demandes de formation des citoyens - notamment dans le cadre du pouvoir et du financement des Régions - sur les offres des organismes, souvent liées aux stratégies des grandes firmes financeuses. Si ces quatre processus se mettaient en marche - ce qui n'est qu'une possibilité parmi d'autres (inertie du système, aménagements à la marge, renforcement de la sélectivité...) - un système rénové se mettrait en place articulant formation professionnelle continue (plan de formation), promotion sociale (congé individuel de formation et en partie le droit individuel à la formation) et éducation permanente (une partie du droit individuel à la formation et surtout la validation des acquis de l'expérience). Le droit conditionnel à la formation finirait par déboucher sur un droit de formation réellement universel. Une vraie avancée.

\section{Bibliographie}

Aventur F. et Hanchane S. (1999), «Inégalités d'accès et pratiques de formation continue dans les entreprises françaises » Formation Emploi n ${ }^{\circ} 66$, pp. 5-20.

Bonnet B. (1999), La Formation Professionnelle des Adultes, l'institution et ses formateurs, Paris, L'Harmattan.

Brucy G., Caillaud P., Quenson E., Tanguy L. (2007), Former pour réformer. Retour sur la formation permanente (1945-2004), Paris, La Découverte.

Brucy G. (2000), « Le système français de formation professionnelle continue : mise en perspective historique », Entreprises et Histoire, ${ }^{\circ}$ 25, pp. 65-86.

Calan D. de et Quentin J.-C. (2006), « Les Accords Nationaux Interprofessionnels de 2003 », in Morvan Y. (Éd.), op. cit., pp. 115-120.

Charlot B. (1997), Du rapport aux savoirs : Éléments pour une théorie, Paris, Anthropos.
Chosson J.-F. (1995), Peuple et Culture 1945-1995, Paris, Éditions PEC.

Correia M. et Pottier F. (2000), « La formation tout au long de la vie $»$ : progression professionnelle ou adaptation aux contraintes?», Formation Emploi, $\mathrm{n}^{\circ} 71, \mathrm{pp} .65-82$.

Dayan J.-L. et Eksi J. (2007), À quoi sert la Formation Professionnelle Continue? Centre d'Analyse Stratégique, Note du Département Travail-Emploi-Formation, reproduit dans Partage, janvier-avril, pp. 72-76.

Debré M. (1960) «La promotion supérieure du travail », Discours de Cachan reproduit dans Le Livre Blanc de la Promotion Sociale, La Documentation française, pp. 122-125.

Delors J. (1991) «Genèse d'une loi et stratégie du changement », Formation Emploi n ${ }^{\circ} 34$, pp. 31-38.

Dubar C. et Engrand S. (1991), « Formation continue et dynamique des identités professionnelles », Formation Emploi $\mathrm{n}^{\circ}$ 34, pp. 87-100. 
Dubar C. et Gadéa C. (Éds) (1999), La promotion sociale en France, Lille, Presses du Septentrion.

Dubar C. (2004), La formation professionnelle continue, Paris, La Découverte, Repères, $5^{\mathrm{e}}$ éd.

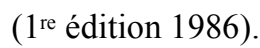

Dubar C. (2006), « Évolution des dispositifs français de formation post-scolaire: l'hypothèse de trois matrices de la formation » in Morgan Y. (Éd.) op. cit., pp. 45-57.

Dumazedier J. (1968), Article «Éducation Permanente » de l'Encyclopédia Universalis.

Dumazedier J. (1996), La Leçon de Condorcet, Paris, L'Harmattan.

Dupray A. et Hanchane S. (2001), « Les effets de la formation continue en entreprise sur la mobilité et les salaires », Formation Emploi n ${ }^{\circ} 73$, pp. 53-73.

Faure E. et alii (1972), Apprendre à être, Paris, Fayard-UNESCO.

Forquin J.-C. (2002) Les composantes doctrinales de l'idée d'éducation permanente, Paris, L'Harmattan, pp. 7-45.

Fougère D., Goux D. et Maurin E. (2001), « Formation continue et carrières salariales. Une évaluation des données individuelles ", Annales d'économie et de statistique, $\mathrm{n}^{\circ} 63$, pp. 13-37.

Fournier C. (2003), «Développer la formation des "seniors". Deux questions préliminaires », Formation Emploi n ${ }^{\circ}$ 81, pp. 37-50.

Fournier C. (2004), « Aux origines de l'inégale appétence des salariés pour la formation », Cereq-Bref, $\mathrm{n}^{\circ} 209$.

Fournier C. (2006), « Les besoins de formation non satisfait des salariés au prisme des catégories sociales », Formation Emploi n ${ }^{\circ}$ 95, pp. 25-40.

Frétigné C. (2007) L'appétence pour la formation. Une entreprise de rationalisation du flou, Paris, Michel Houdiard.
Fritsch P. (1971), L'éducation des adultes, ParisLa Haye, Mouton.

Gadéa C. et Trancart D. (2003), «Pratiques de la formation continue et promotion au statut de cadre : un lien paradoxal », Formation Emploi n ${ }^{\circ}$ 81, pp. 99-11.

Gonnin-Bolo A. (2005), Entreprises et écoles : une rencontre de professionnels, Paris, INRP.

Hanchane S. et Lambert M. (2003), «Variété des modes de formation, usages et enjeux » Formation Emploi $\mathrm{n}^{\circ}$ 81, pp. 51-66.

Hédoux J. (1982), « Des publics et des non-publics de la formation d'adultes. L'accès à l'ACF de Sallaumines - Noyelles-sous-Lens ", Revue française de sociologie, XXIII-2, pp. 76-90.

IFOREP (2004) « La vie pour se former », Numéro commun Le Monde Initiatives et les Cahiers de l'Iforep $\mathrm{n}^{\circ} 112$, octobre-décembre.

Larsson S. (2001), « Les cercles d'études et la démocratie en Suède », Éducation Permanente $\mathrm{n}^{\circ} 149$, pp. 231-57.

Maggi-Germain N. (2006), "Herméneutique juridique de l'ANI de 2003 et de la Loi de $2004 »$, in Morvan Y. (Éd.) op. cit., pp. 103-114.

Maggi-Germain N. (2004), « Entre individualisation et personnalisation des droits des salariés », Droit social I, pp. 43-54.

Méhaut P. (2001), «Gouverner les systèmes de formation professionnelle: planification, marché, coordination », Formation Emploi n ${ }^{\circ} 16$, pp. 224-35.

Méhaut P. (2003), « Repenser la formation continue », Formation Emploi $\mathrm{n}^{\circ}$ 81, pp. 27-35.

Méhaut P. (2006) « Les enjeux futurs de la Formation tout au long de la vie » in Morvan Y. (Éd) op. cit, pp. 163-174.

Merle V. et Lichtenberger Y. (2001) «Formation et éducation tout au long de la vie : deux réformes, un même défi », Formation Emploi n ${ }^{\circ} 76$, pp. 169-190. 
Merle V. (2004), « De l'éducation permanente à la formation tout au long de la vie », Travail et Emploi $\mathrm{n}^{\circ} 100$, pp. 129-153.

Merle V. (2006) «La formation tout au long de la vie : un projet pour les sociétés démocratiques », in Morvan Y. (Éd) op. cit., pp. 23-38.

Möbus M. (2006) «L'articulation formation initiale/ formation continue en France et en Allemagne », in Morvan Y. (Éd) : op. cit., pp. 59-72.

Montlibert C. de (1991), L'institutionnalisation de la formation permanente, Strasbourg, Presses Universitaires.

Morvan Y. (Éd. 2006), La formation tout au long de la vie. Nouvelles questions, nouvelles perspectives, Colloque de Rennes, mars, Presses Universitaires de Rennes.

Podevin G. (1999) «Formation et promotion : un lien démocratique rompu », in Dubar C. et Gadéa C, op. cit, pp. 154-176.

Podevin G. et Verdier É. (1989) Formation continue et compétitivité économique, Paris, Céreq, coll. des Études, $\mathrm{n}^{\circ} 51$.

Podevin G. et Ghaffari S. (Éds) (2008), Les Fongécif, Presses Universitaires de Rennes.
Poullaouec T. (2004) «Les jeunes ouvriers et la formation tout au long de la vie $»$ : promotion ou insertion ? », Formation Emploi n ${ }^{\circ}$ 86, pp. 53-68.

Rose J. (1986), Les jeunes et l'emploi, Économica.

Schwartz B. (1969), «Le concept d'éducation permanente », Éducation permanente $\mathrm{n}^{\circ} 1$, pp. 7-24

Schwartz B. (1994), Moderniser sans exclure, Paris, La Découverte.

Tanguy L. (2007a), «La fabrication d'un bien universel », in Brucy et alii, op. cit., pp. 31-68.

Tanguy L. (2007b), « De la négociation entre interlocuteurs sociaux au dialogue social entre les partenaires », in Brucy et alii, op. cit, pp. 245-265.

Troger V. (2001), «Les passeurs de l'éducation populaire à la formation continue», Travail et Emploi $\mathrm{n}^{\circ}$ 86, pp. 9-24.

Tuchszirer C. (2003), « La nouvelle offre de formation de l'UNEDIC dans le cadre de la mise en place du PARE », DARES : Les évolutions de la formation professionnelle, La Documentation française, pp. 271-282.

Ueberschlag G. et Muller F. (1986), Éducation populaire: objectifs d'hier et d'aujourd'hui, Presses Universitaires de Lille. 
Résumé

\section{Les changements possibles du système français de formation continue}

Claude Dubar

Le système français de formation privilégie une "matrice " liant la formation et le travail, le financement par les entreprises et le modèle des stages courts d'adaptation au détriment d'une part d'une matrice reliant à une promotion sociale des formations longues, de type cours du soir, sur financement public et d'autre part d'une matrice centrée sur le développement culturel, la citoyenneté et l'éducation permanente. Les législations récentes (lois de 2002 et 2004) visent à rééquilibrer le système. Quatre changements possibles sont analysés : accroître la responsabilité des adultes, réduire les inégalités, améliorer le rendement des formations pour les salariés et les chômeurs, donner la priorité aux demandes sur les offres de formation, notamment dans un cadre régional. L'inventaire des conditions de ces changements conduit à un optimisme modéré.

\section{Mots clés}

Formation professionnelle, législation de la formation professionnelle, formation tout au long de la vie, validation des acquis

Journal of Economic Literature: M 53 - Training ; L 22 - Firm Organization and Market Structure 